\title{
Sepsis secundaria a infección urinaria asociada a sonda vesical por Trichosporon asahii en una unidad pediátrica de quemados: reporte de $\mathbf{2}$ casos Trichosporon asahii sepsis associated with urinary catheter in a pediatric burn unit: 2 case reports
}

\author{
Dra. Florencia Escarráa, Dra. Jimena Lema ${ }^{b}$, Bqca. Beatriz Caraccioloc, Bqca. Susana Carnovalec, \\ Lic. Verónica Álvarez ${ }^{d}$, Dra. Nidia Tramontib ${ }^{b}$ Dr. Fabián Guarracino ${ }^{b}$ y Dra. M. Teresa Rosanova ${ }^{a}$
}

\section{RESUMEN}

Trichosporon asahii es un hongo ubicuo que se ha aislado como parte de la microbiota humana. Recientemente, se ha visto una emergencia de este patógeno en infecciones tanto localizadas como sistémicas. En unidades de cuidados intensivos pediátricos para quemados, existen escasos reportes de infecciones del tracto urinario por este microorganismo.

Se describen 2 pacientes pediátricos con internación prolongada por quemaduras extensas y múltiples tratamientos antibióticos previos. Ambos presentaron sepsis por infección del tracto urinario asociada a sonda vesical por Trichosporon asahii.

En ambos pacientes, se realizó el recambio de la sonda vesical y tratamiento con voriconazol por 10 días, con buena evolución. En los casos presentados, debido a la ausencia de otros aislamientos microbiológicos y a la buena respuesta al tratamiento antifúngico junto con el recambio de la sonda vesical, se asumió al Trichosporon asahii como el probable agente causal de la sepsis.

Palabras clave: Trichosporon asahii, infecciones urinarias, unidades de quemados, pediatría.

\begin{abstract}
Trichosporon asahii is a ubiquitous fungus that has been isolated as part of human microbiota. There has been an emergence of this pathogen in recent years, causing superficial and deep seated infections. There are scarce reports of urinary tract infections in pediatric intensive care burn units caused by this agent.

We describe the cases of 2 pediatric patients with prolonged hospitalization due to severe burns that had received several antibiotic courses for previous infections. Both presented sepsis secondary to catheter related urinary tract infection by
\end{abstract}

a. Servicio de Control Epidemiológico e Infectología.

b. Unidad de Cirugía Plástica y Quemados.

c. Servicio de Microbiología.

d. Control de Infecciones.

Hospital de Pediatría "Prof. Dr. Juan P. Garrahan",

Ciudad Autónoma de Buenos Aires, Argentina.

Correspondencia:

Dra. Florencia Escarrá,florescarra@gmail.com

Financiamiento: Ninguno.

Conflicto de intereses: Ninguno que declarar.

Recibido: 7-2-2017

Aceptado: 9-6-2017
Trichosporon asahii. Both patients underwent urinary catheter replacement and were treated effectively with voriconazole for 10 days.

In the cases presented, sepsis was assumed to be due to Trichosporon asahii since no other microorganism was identified and the patients showed favorable outcome with the prescribed treatment with voriconazole and replacement of the urinary catheter.

Key words: Trichosporon asahii, urinary tract infections, burn units, pediatrics.

http: / / dx.doi.org/10.5546/ aap.2017.e311

Cómo citar: Escarrá F, Lema J, Caracciolo B, et al. Sepsis secundaria a infección urinaria asociada a sonda vesical por Trichosporon asahii en una unidad pediátrica de quemados: reporte de 2 casos. Arch Argent Pediatr 2017;115(5):e311-e314.

\section{INTRODUCCIÓN}

Trichosporon asahii (T. asahii) es un hongo ubicuo que se ha aislado como parte de la microbiota humana, especialmente en el área perigenital. ${ }^{1}$

En los últimos años, se ha visto una emergencia de este patógeno en infecciones tanto localizadas como sistémicas en huéspedes inmunocomprometidos, quemados, trasplantados y en tratamiento con corticoides, entre otros. En unidades de cuidados intensivos pediátricos para quemados (UCIQ), existen escasos reportes de infecciones del tracto urinario (ITU) por este microorganismo.

Se presentan 2 pacientes con sepsis por ITU asociada a sonda vesical (SV) por T. asahii documentadas durante el mes de enero de 2016 en una UCIQ.

\section{Caso Nro. 1:}

T. asahii se aisló en 2 oportunidades en la orina de un niño de 2 años que estaba internado por quemadura del $35 \%$ de su superficie corporal (SC) por líquidos calientes. El paciente requirió 
internación prolongada, ventilación asistida, inotrópicos, SV, escarectomías sucesivas y autoinjerto debido a la mala evolución de la quemadura. Como complicaciones, presentó sepsis por Proteus mirabilis, Providencia spp., Acinetobacter baumannii y Staphylococcus aureus meticilino resistente, con tromboflebitis femoral e ilíaca derecha y dificultad respiratoria. En el día 14 de internación, presentó fiebre e hipotensión con requerimiento de inotrópicos. Se encontraba recibiendo tratamiento antibiótico con meropenem, colistín, ampicilina-sulbactam y vancomicina por las infecciones mencionadas anteriormente. Por un cuadro compatible con sepsis, se tomaron hemocultivos, cultivo diferencial de catéter y urocultivo. Al informarse sedimento urinario patológico y presencia de levaduras en dos urocultivos consecutivos, se adicionó anfotericina $B$ desoxicolato al esquema antibiótico. El primer urocultivo se tomó por punción de SV con técnica adecuada, y el segundo, luego de su recambio. Se identificó T. asahii en ambos urocultivos. No presentaba otro foco clínico ni otro aislamiento microbiológico en ese momento.

\section{Caso Nro. 2:}

Paciente de 4 años que requirió internación por quemadura del $60 \%$ de la SC por fuego directo secundario a un incendio de la vivienda, asociado a síndrome inhalatorio. Requirió ventilación asistida, inotrópicos y SV desde su ingreso y tratamiento quirúrgico con escarotomías en 4 miembros. Recibió autoinjertos del $45 \%$ de su SC. Presentó sepsis por Pseudomonas aeruginosa multirresistente e infección de la quemadura por Acinetobacter baumannii multirresistente para los cuales estaba recibiendo tratamiento antibiótico con colistín y meropenem. En el día 26 de internación, presentó un episodio de fiebre e hipotensión arterial compatible con shock séptico. Se tomaron hemocultivos y cultivo diferencial de catéter, que resultaron negativos, y un urocultivo, con informe de sedimento patológico y presencia de levaduras, por lo que se agregó anfotericina B desoxicolato. Se obtuvo desarrollo de T. asahii en el urocultivo tomado por punción de SV con técnica estéril, el cual se repitió luego del recambio de la SV y se obtuvo un nuevo resultado positivo para el mismo germen.

Las muestras de orina obtenidas en muestras sucesivas de urocultivo pretratamiento antifúngico y con recambio de la SV se sembraron para el estudio micológico en agar glucosado de Sabouraud y se incubaron a $37^{\circ} \mathrm{C}$ y $28^{\circ} \mathrm{C}$. Al cabo de 48-72 horas, se observaron colonias características blancas y cremosas de aspecto cerebriforme y que, con el tiempo, se volvieron secas. El estudio microbiológico se realizó mediante el examen en fresco con azul de lactofenol, que evidenció la presencia de artroconidias características de T. asahii.

La identificación se realizó según la capacidad de hidrolizar sustratos cromogénicos en presencia de un indicador (CHROMAgar), según la utilización de hidratos de carbono por API 20C AUX (BioMérieux) y según la producción de compuestos nitrogenados (urea). ${ }^{2}$

Al identificarse el T. asahii, se modificó el tratamiento antifúngico a voriconazol. Ambos pacientes recibieron tratamiento por 10 días, con buena evolución, y, actualmente, se encuentran en seguimiento ambulatorio por las secuelas funcionales y estéticas secundarias a la quemadura.

\section{COMENTARIO}

Los pacientes con quemaduras extensas presentan múltiples factores de riesgo que los predisponen a la colonización y / o infección fúngica, entre ellos, las múltiples cirugías, uso de esteroides, presión antibiótica, nutrición parenteral, profilaxis antifúngica, accesos vasculares, neutropenia, sondaje urinario y ventilación mecánica invasiva. ${ }^{3}$ El riesgo aumenta en aquellos pacientes con quemaduras de más del 30\% de la SC, quemaduras profundas y en aquellos que no han recibido injertos precozmente.

El T. asahii es un germen infrecuente en la Unidad de Quemados, y Candida spp. son los hongos más frecuentes. En una cohorte prospectiva estudiada en la UCIQ del Hospital Garrahan entre 2007 y 2009, se registraron 48 episodios de sepsis. De los agentes fúngicos, Candida spp. fueron los más frecuentes, y se reportaron 2 casos por T. asahii. ${ }^{4}$

Dada su ubicuidad y por ser un colonizante habitual del tracto genitourinario, es difícil atribuirle al T. asahii un rol patógeno. En un estudio realizado por Taverna et al. en Argentina, se identificó el T. asahii como la especie de Trichosporon más frecuente causante de infecciones profundas, y el genotipo más frecuente fue el $1 .^{5}$

La identificación del T. asahii se realiza, generalmente, sobre la base de sus características 
fisiológicas (asimilación de hidratos de carbono, resistencia a la cicloheximida, desarrollo a $37^{\circ} \mathrm{C}$ ) y morfológicas. En el cultivo en agar Sabouraud, se desarrolla formando colonias blancas o cremosas, de aspecto cerebriforme (Figura 1) y se caracteriza por la formación de artroconidias, blastoconidias, hifas y pseudohifas. ${ }^{6}$

La habilidad del T. asahii para formar biofilm en materiales protésicos contribuye a su invasividad, ya que le permite evadir la respuesta inmune del huésped y el tratamiento antifúngico. ${ }^{7}$

La identificación precisa es relevante dada la sensibilidad disminuida del Trichosporon a la anfotericina B. El tratamiento de elección se modificó a lo largo del tiempo. Ya no se recomienda la anfotericina $\mathrm{B}$ porque mostró pobre respuesta y fallas de tratamiento. Las equinocandinas, dada la concentración inhibitoria mínima (CIM) alta del T. asahii a este grupo, no constituyen una opción $\mathrm{y}$, además, se documentaron infecciones intratratamiento con ellas. ${ }^{8}$

Si bien comités internacionales, como el Clinical Laboratory Standards Institute(CLSI) y European Committee on Antimicrobial Susceptibility

FIguRA 1. Desarrollo de Trichosporon asahii en la muestra de orina del paciente del caso 1

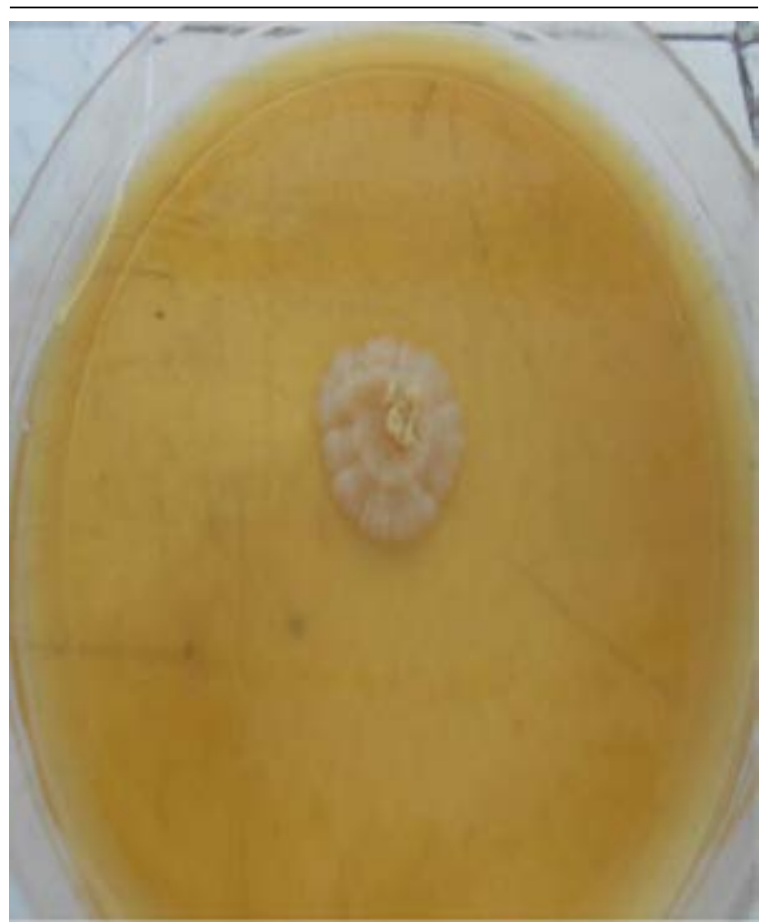

Cultivo de Trichosporon asahii en agar Sabouraud. Colonias blancas o cremosas de aspecto cerebriforme.
Testing (EUCAST), no establecieron, a la fecha, puntos de corte que permitieran determinar el perfil de sensibilidad para T. asahii, varios son los trabajos que demuestran que los valores de CIM para anfotericina son mayores que para las drogas azólicas, que constituyen el tratamiento de elección. Entre estas últimas, diversos autores coinciden en que los valores más bajos de CIM corresponderían a voriconazol. ${ }^{4,9,10}$ Este es un antifúngico triazólico utilizado en el tratamiento de Aspergillus spp., Candida spp., Fusarium spp., Scedosporium spp.y otros hongos. Sus vías de administración son oral y endovenosa. En pediatría, se recomienda una dosis de carga de $9 \mathrm{mg} / \mathrm{kg} /$ dosis cada $12 \mathrm{~h}$ y luego dosis de mantenimiento de $8 \mathrm{mg} / \mathrm{kg} /$ dosis cada $12 \mathrm{~h}$ con el fin de alcanzar niveles plasmáticos $\geq 1-2 \mu \mathrm{g} / \mathrm{ml}$. Habitualmente, es bien tolerado; los principales efectos adversos son alteraciones visuales dependientes de la dosis, reversibles, como visión borrosa, cambios en la percepción de los colores y/o fotofobia. También se describen aumento en transaminasas hepáticas y reacciones cutáneas, como fotosensibilidad. ${ }^{11}$

Se describen, en la bibliografía, ITU por T. asahii como infección nosocomial en pacientes en la Unidad de Cuidados Intensivos (UCI) con comorbilidades graves, presencia de SV e infecciones bacterianas concomitantes que requirieron uso de antimicrobianos de amplio espectro. ${ }^{12,13}$

La mortalidad descrita para las ITU por T. asahii en algunas series de adultos hospitalizados es del $20-40 \%$, mientras que, para las formas invasivas, asciende a $66-83 \%$, mayor en pacientes neutropénicos o pretérmino. ${ }^{12,14,15}$

Dada su elevada morbimortalidad y que puede preceder a una infección invasiva, es importante mantener un alto índice de sospecha para realizar un correcto diagnóstico y jerarquizar su presencia en las muestras clínicas de los pacientes, especialmente en quemados.

A modo de conclusión, el T. asahii es un hongo infrecuente en la Unidad de Quemados.

En los casos presentados, debido a la ausencia de otros aislamientos microbiológicos y a la buena respuesta al tratamiento antifúngico junto con el recambio de SV, se lo asumió como el probable agente causal de la sepsis en ambos pacientes. El voriconazol fue efectivo en ambos pacientes.

\section{Agradecimientos}

A todo el equipo de la Unidad Pediátrica de Quemados del Hospital "Juan P. Garrahan". 


\section{REFERENCIAS}

1. Tamayo Lomas L, Domínguez-Gil González M, Martín Luengo AI, et al. Infección nosocomial por Trichosporon asahii en un paciente quemado crítico. Rev Iberoam Micol 2015;32(4):257-60.

2. Biasoli MS, Carlson D, Chiganer GJ, et al. Systemic infection caused by Trichosporun asahii in a patient with liver transplant. Med Mycol 2008;46(7):719-23.

3. Ha JF, Italiano CM, Heath $\mathrm{CH}$, et al. Candidemia and invasive candidiasis: a review of the literature for the burns surgeon. Burns 2011;37(2):181-95.

4. Rosanova MT, Stamboulian D, Lede R. Infecciones en los niños quemados: análisis epidemiológicoy de los factores de riesgo. Arch Argent Pediatr 2013;111(4):303-8.

5. Taverna CG, Córdoba S, Murisengo OA, et al. Molecular identification, genotyping and antifungal susceptibility testing of clinically relevant Trichosporon species from Argentina. Med Mycol 2014;52(4):356-66.

6. Carnovale S, Guelfand L. Trichosporon asahii. Rev Argent Microbiol 2012;44(3):231.

7. Di Bonaventura G, Pompilio A, Picciani C, et al. Biofilm formation by the emerging fungal pathogen Trichosporon asahii: development, architecture, and antifungal resistance. Antimicrob Agents Chemother 2006;50(10):3269-76.

8. Liao $\mathrm{Y}$, HartmannT, Zheng $\mathrm{T}$, et al. Break through trichosporon osisin patients receiving echinocandins: case report and literature review. Chin Med J (Engl) 2012;125(14):2632-5.
9. Hazirolan G, Canton E, Sahin S, et al. Head-to-head comparison of inhibitory and fungicidal activities of fluconazole, itraconazole, voriconazole, posaconazole, and isavuconazole against clinical isolates of Trichosporon asahii. Antimicrob Agents Chemother 2013;57(10):4841-7.

10. Mekha N,Sugita T, Ikeda R, et al. Genotyping and antifungal drug susceptibility of the pathogenic yeast Trichosporon asahii isolated from Thai patients. Mycopathologia 2010;169(1):67-70.

11. Steinbach WJ, Cohen-Wolkowiez M, Benjamin DK. Principios de tratamiento antifúngico. In: Kliegman R, Stanton B, Geme J, et al. Nelson Textbook of Pediatrics. $20^{\mathrm{a}}$ ed. Philadelphia: Elsevier; 2016.p.1512-5.

12. Silva V, Zepeda G, Alvarado D. Infección urinaria nosocomial por Trichosporon asahii. Primeros dos casos en Chile. Rev Iberoam Micol 2003;20(1):21-3.

13. MattedeMd, Piras C, Mattede KD, et al. Urinary tract infections due to Trichosporon spp. in severely ill patients in an intensive care unit. RevBras Ter Intensiva 2015;27(3): 247-51.

14. Rodrigues GdaS, Faria RR, Guazzelli LS, et al. Infección nosocomial por Trichosporon asahii: revisión clínica de 22 casos. Rev Iberoam Micol 2006;23(2):85-9.

15. Vashishtha VM, Mittal A, Garg A. A fatal outbreak of Trichosporon asahii sepsis in a neonatal intensive care Unit. Indian Pediatr 2012;49(9):745-7. 